\title{
A Liability Tracking Approach to Long Term Management of Pension Funds
}

\author{
Masashi Ieda ${ }^{1}$, Takashi Yamashita ${ }^{2}$, Yumiharu Nakano ${ }^{1}$ \\ ${ }^{1}$ Graduate School of Innovation Management, Tokyo Institute of Technology, Tokyo, Japan \\ ${ }^{2}$ The Government Pension Investment Fund, Tokyo, Japan \\ Email: ieda@craft.titech.ac.jp
}

Received May 28, 2013; revised July 2, 2013; accepted July 11, 2013

Copyright (C) 2013 Masashi Ieda et al. This is an open access article distributed under the Creative Commons Attribution License, which permits unrestricted use, distribution, and reproduction in any medium, provided the original work is properly cited.

\begin{abstract}
We propose a long term portfolio management method which takes into account a liability. Our approach is based on the LQG (Linear, Quadratic cost, Gaussian) control problem framework and then the optimal portfolio strategy hedges the liability by directly tracking a benchmark process which represents the liability. Two numerical results using empirical data published by Japanese organizations are served: simulations tracking an artificial liability and an estimated liability of Japanese organization. The latter one demonstrates that our optimal portfolio strategy can hedge his or her liability.
\end{abstract}

Keywords: Pension Fund Management; Long Term Portfolio Optimization; Quadratic Hedging; Stochastic Optimal Control; Hamilton-Jacobi-Bellman Equations; LQG Control

\section{Introduction}

In the management of pension funds, a long term portfolio strategy taking into account a liability is one of the most significant issues. The main reason is the demographic changes in the developed countries: if the working-age population is enough to provide for old age, the liability is a minor issue in the portfolio management of pension funds. Since the life expectancy has increased in recent decades, it becomes insufficient to provide for old age. Furthermore, the low birth rate continues and drives up this problem for decades. Thus pension funds face a challenging phase to construct long term portfolio strategies which hedge their liabilities.

A lot of pension funds except a few ones [1] determine their portfolio strategies by the traditional single time period mean variance approach which excludes an evaluation of a liability. Its intuitive criterion attracts managers of pension funds. However the single time period approach is unsuitable for a long term portfolio management in the sense that it is unable to change the strategy excepting the initial time. The multi time period approach which arrows the change of the strategy has a problem that the computational complexity grows exponentially. Hence if we employ this approach, we are usually unable to obtain the optimal portfolio strategy in realistic time.
Therefore the aim of this paper is to propose a long term portfolio strategy which 1) involves an evaluation of a liability, 2) admits changes of the strategy at any time, and 3) is obtained in realistic time. To tackle this problem, we employ the LQG (Linear, Quadratic cost, Gaussian) control problem (see, e.g., Fleming and Rishel [2]). The LQG control problem is a class of stochastic control problem and is able to provide the control minimizing the mean square error of a benchmark process and a controlled process. Roughly speaking our tactic is that we compute the optimal portfolio strategy with the benchmark process which represents the liability. Then we can track the liability by using our optimal portfolio strategy. Although it is difficult to obtain the solution of stochastic control problem in general, the LQG control problem has the analytical solution which assures that we are able to obtain the solution in realistic time and thus it meets our purpose.

A continuous time stochastic control approach is one of the most popular methods to obtain the suitable long term portfolio strategy. The literature about this approach is quite rich. The papers treating the management of pension funds are, for instance, as follows: Deelstra et al. [3] and Giacinto et al. [4] discuss the portfolio management for pension funds with a minimum guarantee; Menoncin and Scaillet [5] and Gerrard et al. [6] deal with the pen- 
sion scheme including the de-cumulation phase. Our stu$d y$ is on the cutting edge in the sense that deals with tracking liabilities directly and constructs a suitable long term portfolio at the same time.

The organization of the present paper is as follows. We introduce continuous time models of assets and a benchmark in Section 2. To fit in the LQG control problem, they are defined by the linear stochastic differential equations (SDEs). We mention that our portfolio strategy is represented by the amounts of assets. In Section 3, we define a criterion of the investment performance and provide the optimal portfolio strategy explicitly. Several numerical results are served in Section 4 Throughout the section the parameters related to the assets are determined by an empirical data provided by the Government Pension Investment Fund in Japan. The simulation using an artificial data is discussed in Section 4.1 and this result gives conditions that our optimal portfolio strategy works well. Section 4.2 provides a case study using an empirical estimation published by the Japanese Ministry of Health, Labour and Welfare. It demonstrates that our strategy is able to hedge the liability well.

\section{Continuous Time Models of Assets and a Benchmark}

In this section, we present mathematical models of assets and a benchmark. The market which we are considering consists of only one risk-free asset and $n$-risky assets and we have $m$-benchmark component processes.

Let $\left(\Omega, \mathcal{F},\left\{\mathcal{F}_{t}\right\}_{t \geq 0}, \mathbb{P}\right)$ be a filtered probability space $\left\{W_{t}\right\}_{t \geq 0}$ be a $d$-dimensional Brownian motion where $d=n+m$ and $\mathcal{L}^{2}\left(\mu_{T} \times \mathcal{L}\right)$ be a space of stochastic processes $\left\{Z_{t}\right\}_{t \geq 0}$ which satisfy

$$
\mathbb{E}\left[\int_{0}^{T}\left|Z_{t}\right|^{2} \mathrm{~d} t\right]<\infty .
$$

We denote price process of the risk-free asset, those of the risky assets and the benchmark component processes by $S_{t}^{0}, S_{t}=\left(S_{t}^{1}, \cdots, S_{t}^{n}\right)^{*}$ and $Y_{t}=\left(Y_{t}^{1}, \cdots, Y_{t}^{m}\right)$ respectively, where the asterisk means transposition. To fit in the LQG control problem, we assume that $S_{t}^{0}, S_{t}$ and $Y_{t}$ are governed by the following SDEs:

$$
\begin{aligned}
& \left\{\begin{array}{l}
\frac{\mathrm{d} S_{t}^{0}}{S_{t}^{0}}=r(t) \mathrm{d} t, \\
S_{0}^{0}=S_{0} \in \mathbb{R},
\end{array}\right. \\
& \left\{\begin{array}{l}
\frac{\mathrm{d} S_{t}^{i}}{S_{t}^{i}}=b^{i}(t) \mathrm{d} t+\sum_{j=1}^{d} \sigma_{S}^{i j}(t) \mathrm{d} W_{t}^{j}, i=1,2, \cdots, n, \\
S_{0}^{i}=s_{0}^{i} \in \mathbb{R},
\end{array}\right. \\
& \left\{\begin{array}{l}
\mathrm{d} Y_{t}=\left(\alpha(t) Y_{t}+h(t)\right) \mathrm{d} t+\sigma_{Y}(t) \mathrm{d} W_{t}, \\
Y_{0}=y_{0} \in \mathbb{R}^{m},
\end{array}\right.
\end{aligned}
$$

where $r:[0, T] \rightarrow \mathbb{R}, b:[0, T] \rightarrow \mathbb{R}^{n}, \sigma_{S}:[0, T] \rightarrow \mathbb{R}^{n \times d}$, $\alpha:[0, T] \rightarrow \mathbb{R}^{m \times m}, h:[0, T] \rightarrow \mathbb{R}^{m}$ and

$\sigma_{Y}:[0, T] \rightarrow \mathbb{R}^{m \times d}$ are deterministic continuous functions and $T<\infty$ represents the maturity. Coefficients $r, b^{i}$ and $\sigma_{s}$ stand for the risk-free rate and the expected return rate of the $i$-th asset and the volatility.

Let a class of portfolio strategy $\mathcal{A}$ be the collection of $\mathbb{R}^{n}$-valued $\mathcal{F}_{t}$-adapted process $\left\{u_{t}\right\}_{0 \leq t \leq T}$ which satisfies

$$
\mathbb{E}\left[\int_{0}^{T}\left|u_{t}\right|^{2} \mathrm{~d} t\right]<\infty
$$

$\xi_{t} \in \mathbb{R}^{n}$ be the amount of the risky asset held by an investor at time $t$, and $X_{t}$ be the value of our portfolio at time $t$. Then the amount of the risk-free asset held by the investor is represented by $X_{t}-\sum_{i=1}^{n} \xi_{t}^{i}$. Hence, $\left\{X_{t}\right\}_{0 \leq t \leq T}$ is governed by

$$
\left\{\begin{array}{l}
\mathrm{d} X_{t}=\sum_{i=1}^{n} \xi_{t}^{i} \frac{\mathrm{d} S_{t}^{i}}{S_{t}^{i}}+\left(X_{t}-\sum_{i=1}^{n} \xi_{t}^{i}\right) \frac{\mathrm{d} S_{t}^{0}}{S_{t}^{0}},\left\{\xi_{t}\right\}_{0 \leq t \leq T} \in \mathcal{A}, \\
X_{0}=x_{0}=s_{0}^{0}+s_{0}^{*} \mathbf{1},
\end{array}\right.
$$

where $\mathbf{1}=(1, \cdots, 1)^{*} \in \mathbb{R}^{n}$. To emphasize the initial wealth and the control variable, we may write $X_{t}=X_{t}^{x_{0}, \xi}$.

The solution $X_{t}$ of the SDE (4) is given as follow:

$$
\begin{aligned}
& X_{t}=\mathrm{e}^{\int_{0}^{t} r(u) \mathrm{d} u} x_{0}+\int_{0}^{t} \mathrm{e}^{\int_{s}^{t} r(u) \mathrm{d} u}(b(s)-r(s) \mathbf{1})^{*} \xi_{s} \mathrm{~d} s \\
& +\int_{0}^{t} \mathrm{e}^{\int_{s}^{t} r(u) \mathrm{d} u} \xi_{s}^{*} \sigma_{S}(s) \mathrm{d} W_{s} .
\end{aligned}
$$

Moreover since $r, b$, and $\sigma_{S}$ are continuous functions on $[0, T]$ and $\xi \in \mathcal{A}, X_{t}$ is in $\mathcal{L}^{2}\left(\mu_{T} \times \mathbb{P}\right)$ :

$$
\begin{aligned}
& \mathbb{E}\left[\int_{0}^{T} X_{t}^{2} \mathrm{~d} t\right] \leq \mathbb{E}\left[\int_{0}^{T}\left(\mathrm{e}^{\int_{0}^{t} r(u) \mathrm{d} u} x_{0}\right)^{2} \mathrm{~d} t\right] \\
& +\mathbb{E}\left[\int_{0}^{T}\left(\int_{0}^{t} \mathrm{e}^{\int_{s}^{t} r(u) \mathrm{d} u}(b(s)-r(s) \mathbf{1})^{*} \xi_{s} \mathrm{~d} s\right)^{2} \mathrm{~d} t\right] \\
& +\mathbb{E}\left[\int_{0}^{T}\left(\int_{0}^{t} \mathrm{e}^{\int_{s}^{t} r(u) \mathrm{d} u} \xi_{s}^{*} \sigma_{S}(s) \mathrm{d} W_{s}\right)^{2} \mathrm{~d} t\right] \\
& \leq T K_{0} x_{0}^{2}+T^{2} K_{1} \mathbb{E}\left[\int_{0}^{T} \xi_{s}^{*} \mathbf{1 1}^{*} \xi_{s} \mathrm{~d} s\right] T K_{2} \mathbb{E}\left[\int_{0}^{T} \xi_{s}^{*} \mathbf{1 1}^{*} \xi_{s} \mathrm{~d} s\right] \\
& <\infty
\end{aligned}
$$

where $K_{0}, K_{1}$ and $K_{2}$ are constants.

\section{Optimal Investment Strategy}

We define the criterion of investment performance $J$ by

$$
\begin{aligned}
J[\xi]= & \mathbb{E}\left[\int_{0}^{T} \gamma_{1}\left(a(t)^{*} Y_{t}-X_{t}^{x_{0}, \xi}\right)^{2} \mathrm{~d} t\right. \\
& \left.+\gamma_{2}\left(A^{*} Y_{T}-X_{T}^{x_{0}, \xi}\right)^{2}\right], \xi \in \mathcal{A},
\end{aligned}
$$


where $\gamma_{1} \geq 0$ and $\gamma_{2} \geq 0$ are constants, $A \in \mathbb{R}^{m}$ is a constant vector, and $a:[0, T] \rightarrow \mathbb{R}^{m}$ is a deterministic continuous function. Hence our investment problem is to find the control $\hat{\xi}$ s.t. $J[\hat{\xi}] \leq J[\xi], \quad \xi \in \mathcal{A}$. Since the performance criterion is represented by quadratic functions, our investment problem becomes the LQG control problem. We determine $a(t), A_{T}$ and the parameters of $Y_{t}$ to be able to regard $a(t)^{*} Y_{t}$ and $A_{T}^{*} Y_{T}$ as a liability.

The optimal portfolio strategy is represented in the following form:

Theorem 1 We define the portfolio strategy $\hat{\xi}$ as follows:

$$
\begin{aligned}
\hat{\xi}_{t} & =\frac{-1}{2 F^{00}(t)}\left(\sigma_{S}(t) \sigma_{S}(t)^{*}\right)^{-1} \\
& \times[(b(t)-r(t) \mathbf{1}) \\
& \cdot\left(2 F^{00}(t) X_{t}+2 \tilde{F}^{0^{*}}(t) Y_{t}+G^{0}(t)\right) \\
& \left.+2 \sigma_{S}(t) \sigma_{Y}^{*}(t) \tilde{F}^{0}(t)\right]
\end{aligned}
$$

where $F^{00}, G^{0}:[0, T] \rightarrow \mathbb{R}$ and $\tilde{F}^{0}:[0, T] \rightarrow \mathbb{R}^{m}$ are solutions of following ordinary differential equations $(O D E s)$ :

$$
\begin{aligned}
& \left\{\begin{array}{l}
\frac{\mathrm{d}}{\mathrm{d} t} F^{00}+\gamma_{1}+2 r(t) F^{00} \\
-\bar{b}(t)^{*}\left(\sigma_{S}(t) \sigma_{S}(t)^{*}\right)^{-1} \bar{b}(t) F^{00}=0, \\
F^{00}(T)=\gamma_{2},
\end{array}\right. \\
& \left\{\begin{array}{l}
\frac{\mathrm{d}}{\mathrm{d} t} \tilde{F}^{0}(t)-\gamma_{1} a(t)+r(t) \tilde{F}^{0}(t)+\alpha(t)^{*} \tilde{F}^{0}(t) \\
-\bar{b}(t)^{*}\left(\sigma_{S}(t) \sigma_{S}(t)^{*}\right)^{-1} \bar{b}(t) \tilde{F}^{0}(t)=0, \\
\tilde{F}^{0}(T)=-2 \gamma_{2} A,
\end{array}\right. \\
& \left\{\begin{array}{l}
\frac{\mathrm{d}}{\mathrm{d} t} G^{0}(t)+r(t) G^{0}(t)+2 h(t)^{*} \tilde{F}^{0}(t) \\
-\bar{b}(t)^{*}\left(\sigma_{S}(t) \sigma_{S}(t)^{*}\right)^{-1} \bar{b}(t) G^{0}(t) \\
-\bar{b}(t)^{*}\left(\sigma_{S}(t) \sigma_{S}(t)^{*}\right)^{-1} \sigma_{S}(t) \sigma_{Y}^{*}(t) \tilde{F}^{0}(t)=0, \\
G^{0}(T)=0 .
\end{array}\right.
\end{aligned}
$$

Here we have written $\bar{b}(t)=b(t)-r(t) \mathbf{1}$.

Then $\hat{\xi}$ satisfies $\hat{\xi} \in \mathcal{A}$ and $J[\hat{\xi}] \leq J[\xi], \quad \xi \in \mathcal{A}$.

The proof of Theorem 1 is given in the appendix.

We note that $\hat{\xi}_{t}$ has feedback terms of $X_{t}$ and $Y_{t}$. This implies that our optimal strategy has delays to catch up the the benchmark process $a(t)^{*} Y_{t}$. Hence the pref- erable situation applying our strategy is the case that $a(t)^{*} Y_{t}$ does not fluctuate violently.

\section{Numerical Results}

We apply our method to an empirical data provided by the Japanese organizations. This section is divided to two subsections according to the type of liabilities: an artificial liability and the liability constructed by the estimations published by the Ministry of Health, Labour and Welfare of Japan. The former one suggests the situation that our optimal strategy works well and the latter one demonstrates that our portfolio strategy is able to hedge the liability.

Before we move on the each subsection, we determine the common parameters in following subsections. The first task is to determine the parameters relating to the benchmark component processes. They consist of the income of a pension fund $C_{t}$ and his or her expense $B_{t}$ and thus $n=2$ and $Y_{t}=\left(C_{t}, B_{t}\right)^{*}$. We set the parameters constructing the benchmark process as follows:

$$
\gamma_{1}=\gamma_{2}=1, a(t)=(-1,1)^{*}, A=(-1,1)^{*} .
$$

Hence, the benchmark process is $B_{t}-C_{t}$ which represents a shortfall of the income and then we regard this shortfall as the liability. To discuss the performance of the strategy, we introduce a hedging error function of the $i$-th sample path $E_{t}^{i}$ and its average $\bar{E}_{t}$ as follows:

$$
E_{t}^{i}=\left|\left(B_{t}-C_{t}\right)-X_{t}^{i}\right|, \bar{E}_{t}=\sum_{i=1}^{N} \frac{E_{t}^{i}}{N},
$$

where $X_{t}^{i}$ is the $i$-th sample path of $X_{t}$ and $N \in \mathbb{N}$ is the number of the sample paths. We set $N=1000$ except as otherwise noted.

The next task is to determine the risk-free rate and the expected return rates and volatilities of risky assets. We invest the following four assets: indices of the domestic bond, the domestic stock, the foreign bond and the foreign stock; we number them sequentially. According to the estimations of return rate and volatilities by the Government Pension Investment Fund in Japan [7], we construct $b(t)$ and $\sigma_{S}(t)$ as follows: $b^{1}(t)=3 \%$,

$$
\begin{aligned}
b^{2}(t)=4.8 \%, & b^{3}(t)=3.5 \% \text { and } b^{4}(t)=5.0 \% ; \\
\sigma_{S}^{i j}(t) & = \begin{cases}\tilde{\Sigma}_{i j} & i, j \in\{1, \cdots, n\}, \\
0 & \text { otherwise, }\end{cases}
\end{aligned}
$$

where $\tilde{\Sigma}$ the Cholesky decomposition of $\Sigma$, a variance-covariance matrix of the assets:

$$
\Sigma=\left(\begin{array}{cccc}
29.7 & 18.2 & -4.39 & -5.41 \\
18.2 & 495 & -77.8 & 119 \\
-4.39 & -77.8 & 181 & 147 \\
-5.41 & 119 & 147 & 394
\end{array}\right) \times 10^{-4}
$$


We choose a money market account as the risk-free asset and we set $r(t)=0.0 \%$.

\subsection{Simulation with an Artificial Liability}

In this subsection, we consider the following an artificial deterministic liability model:

$$
\begin{aligned}
& \left\{\begin{array}{l}
\mathrm{d} C_{t}=0.01 C_{t} \mathrm{~d} t, \\
C_{0}=80[\text { trillion yen], }
\end{array}\right. \\
& \left\{\begin{array}{l}
\mathrm{d} B_{t}=0.01 B_{t} \mathrm{~d} t, \\
B_{0}=100[\text { trillion yen], }
\end{array}\right.
\end{aligned}
$$

i.e., we set $\alpha^{i j}(t)=0.01 \delta_{i j}$ and $h(t)=0$. We assume that our wealth coincides with the benchmark at the initial time: $X_{0}=B_{0}-C_{0}$. We construct the optimal portfolio strategy over three decades, i.e., $T:=30$. Then we determine the functions $F^{00}, \tilde{F}^{0}$ and $G^{0}$ by solving the ODEs (7)-(9) numerically. and simulate $N$ paths of $\left(S_{t}, Y_{t}\right)$ on $[0, T]$ according to Equations (1)-(3) using a standard Euler-Maruyama scheme with time-step $\Delta t=0.25$. Figure 1 describes an investment result of a sample path. The black and red lines in Figure 1 represent $B_{t}-C_{t}$ and $X_{t}$ respectively.

The most significant issue it indicates is that the performance of the strategy is quite poor near the maturity. Figure 2 describing $\bar{E}_{t}$ implies that this poor performance does not depend on the sample path. Figure 3 suggests a key factor of this phenomenon: values of functions $F^{00}, \tilde{F}^{0}$ and $G^{0}$ change drastically between $t=25$ and $t=30$; this time period coincides with the term the hedging error becomes large rapidly. Figure 3 also implies that the existence of the stationary solutions of the ODEs (7)-(9). As described in Figure 2, the strategy relatively works well on the time period when the functions $F^{00}, \tilde{F}^{0}$ and $G^{0}$ reach the stationary state. Hence the strategy will be improved by using the stationary solutions of the ODEs (7)-(9) on entire region.

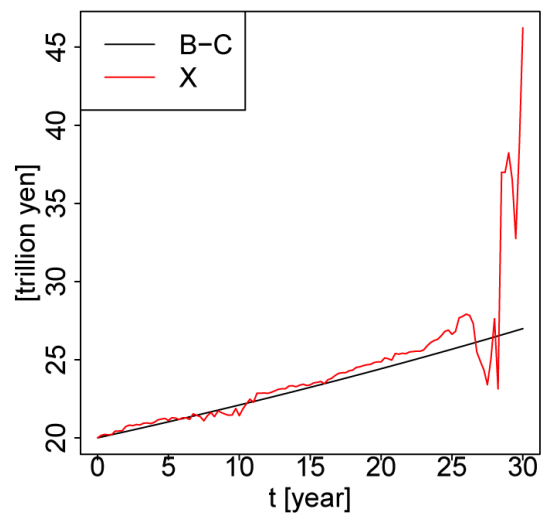

Figure 1. A sample path of $B_{t}-C_{t}$ and $X_{t}$. The black and red lines represent $B_{t}-C_{t}$ and $X_{t}$ respectively.

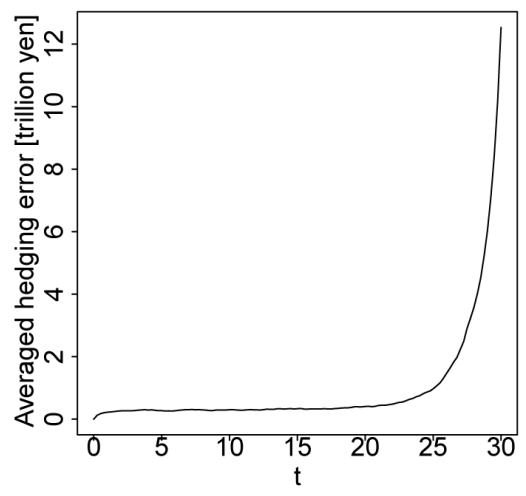

Figure 2. Averaged hedging error $\bar{E}_{t}$.

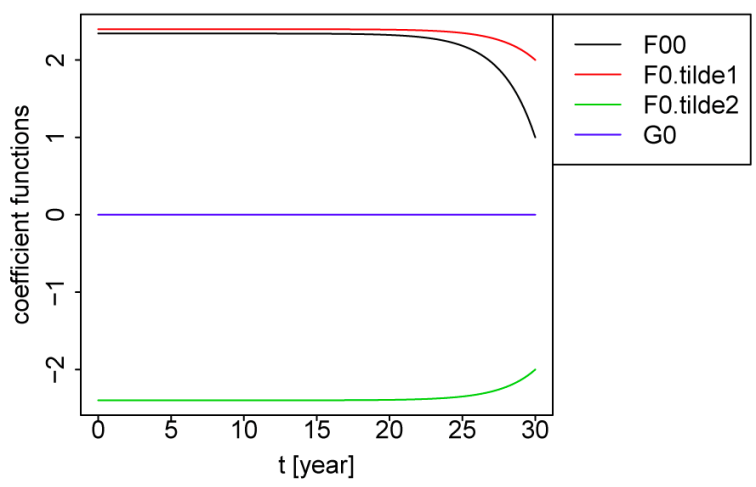

Figure 3. The time evolution of $F^{00}, \tilde{F}^{0}$ and $G^{0}$. The black, red, green and blue lines represent $F^{00}, \tilde{F}^{01}, \tilde{F}^{02}$ and $G^{0}$ respectively.

To obtain the stationary solutions of the ODEs (7)-(9), we replace $T$ to a value large enough. We denote it by $\tilde{T}$ and set $\tilde{T}=50$. Figure 4 shows values of $F^{00}, \tilde{F}^{0}$ and $G^{0}$ obtained by solving the ODEs (7)-(9) with parameter $\tilde{T}$. We can find that the functions $F^{00}, \tilde{F}^{0}$ and $G^{0}$ take the stationary solutions on $[0, T]$.

Results of simulations using the improved strategy are described as follows.

Figures $\mathbf{5}$ and $\mathbf{6}$ indicate that the performance near the maturity is improved and it does not depend on the sample paths. This result leads us to the conclusion that we should construct the strategy with the stationary solutions of the functions $F^{00}, \tilde{F}^{0}$ and $G^{0}$ if they exists.

At the end of this subsection, we mention about our portfolio composition. Figure 7 displays the asset allocation on the sample path described in Figure 5. The money market account, the domestic bond and the for- eign stock indicated by light blue, black and blue lines respectively dominate our portfolio. The optimal strategy is that we keep the most part of the wealth as the money market account and compensate for the increment of the benchmark by the investment for the domestic bond, low risk and low return asset, and the foreign stock, high risk 


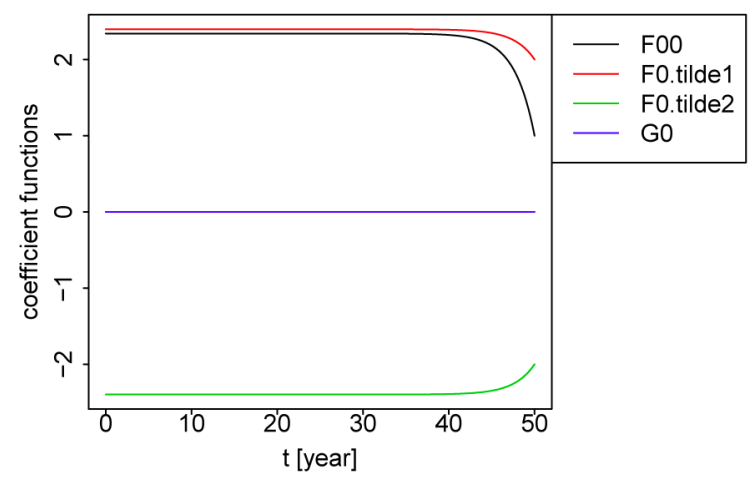

Figure 4. The time evolution of $F^{00}, \tilde{F}^{0}$ and $G^{0}$ (improved case). The black, red, green and blue lines represent $F^{00}, \tilde{F}^{01}, \tilde{F}^{02}$ and $G^{0}$ respectively.

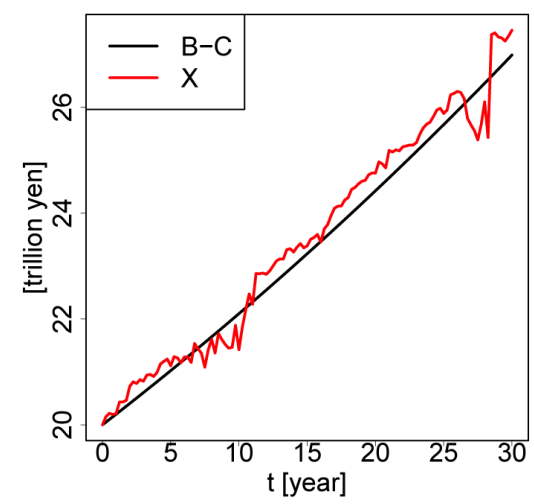

Figure 5. A sample path of $B_{t}-C_{t}$ and $X_{t}$ (improved version). The black and red lines represent $B_{t}-C_{t}$ and $X_{t}$ respectively.

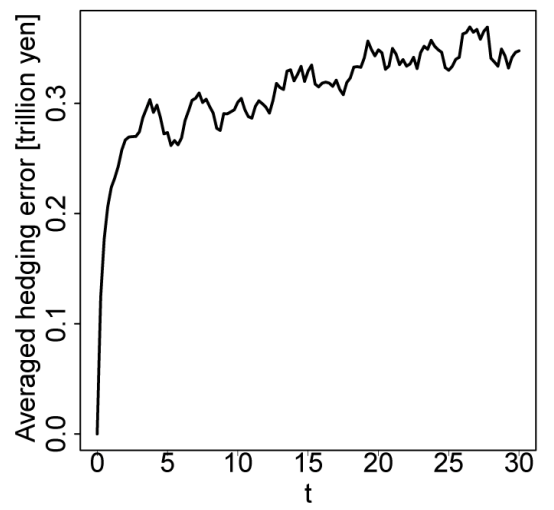

Figure 6. Averaged hedging error $\bar{E}_{t}$ (improved version).

and high return asset. If $X_{t}$ is deficient in $B_{t}-C_{t}$, the strategy increases the proportion of the domestic bond and the foreign stock.

\subsection{Simulation with an Empirical Liability}

According to the Japanese actuarial valuation published in 2009 [8], the estimated income and expense of the welfare pension are showed in the Figure 8.

We regard these estimations as $C_{t}$ and $B_{t}$ and simulate the three decades investments using our optimal strategy from 2040 when the shortfall of the pension fund starts to expand drastically. The following reasons support that this situation is a valid case study: 1) a phase expanding $B_{t}-C_{t}$, the shortfall of the pension fund, is the most typical one expressing the demographic changes; 2) the behaviour of $B_{t}-C_{t}$ in this term meets the condition to apply our optimal strategy: $B_{t}-C_{t}$ is increasing in the entire region. Throughout this subsection we set the start point as the year 2040, i.e., $t=0$ and $t=15$ represent the year 2040 and the year 2055 respectively.

To construct the optimal strategy, we first calibrate $\alpha(t), \sigma_{Y}(t)$ and $h(t)$ to fit the estimations. Setting $\alpha(t)=\sigma_{Y}(t)=0$ and $h(t)$ as a numerical differentiation of the estimations is a simple method to accomplish the purpose. Since we are discussing the three decades portfolio, we determine $T=30$. As suggested in Section

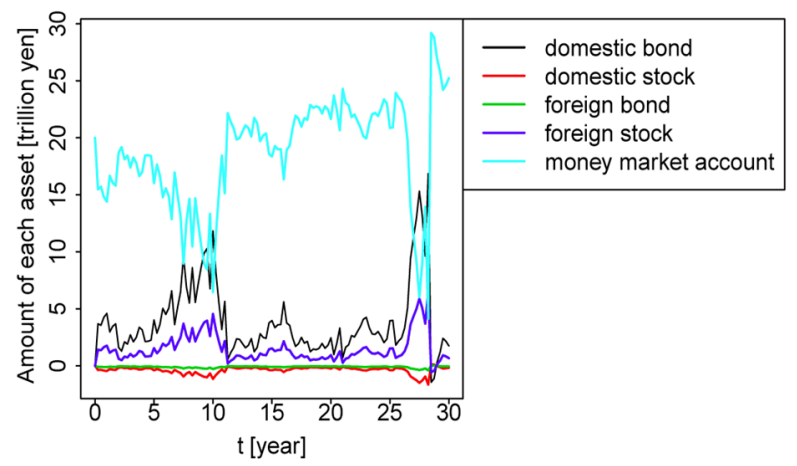

Figure 7. An amount of each asset on the sample path described in Figure 5. The black, red, green, blue and light blue lines represent the amount of a domestic bond, a domestic stock, a foreign bond, a foreign stock and money market account respectively.

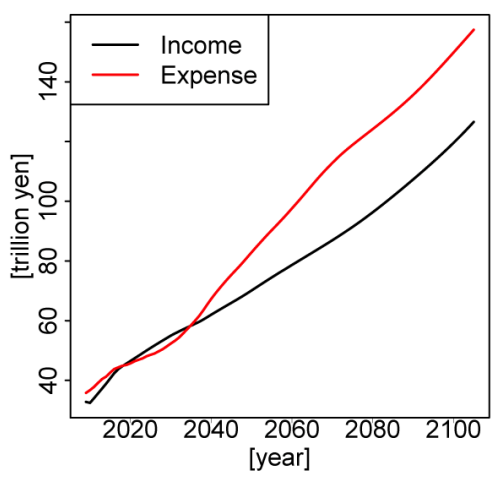

Figure 8. Estimations of the income and the expense of the Japanese welfare pensions. The black and red lines represent estimations of their income and the expense respectively. 
4.1, we set $\tilde{T}=50$ to obtain the stationary $F^{00}$ and $\tilde{F}^{0}$. We are unable to expect the stationary $G^{0}$ because $h(t)$ explicitly depends on $t$. We assume that our wealth coincide with the benchmark at the initial time: $X_{0}=B_{0}-C_{0}$. Then we simulate $N$ paths of $\left(S_{t}, Y_{t}\right)$ on $[0, T]$ according to Equations (1)-(3) using a standard Euler-Maruyama scheme with time-step $\Delta t=0.25$ which means that we can rearrange our portfolio every quarter. Results of the simulations are as follows.

We are able to argue that our strategy hedges the shortfall well since Figure 9 suggests that $\bar{E}_{t}$, the averaged hedging error, is approximately $3 \%$ of $B_{t}-C_{t}$, the shortfall, in every quarter.

Figure 10 displays the asset allocation on the sample path described in Figure 11. In the same manner as in the case of the artificial liabilities discussed in Section 4.1, our optimal portfolio is dominated by the money market account, the domestic bond and the foreign stock. However the proportion of the domestic bond and the foreign stock is much higher. We can understand this phenomenon intuitively: since the shortfall increases more rapid than that discussed in Section 4.1, the hedging portfolio is rearranged to become more profitable. The practical suggestion from this fact is that we have to take a risk to track the increasing liability and this is

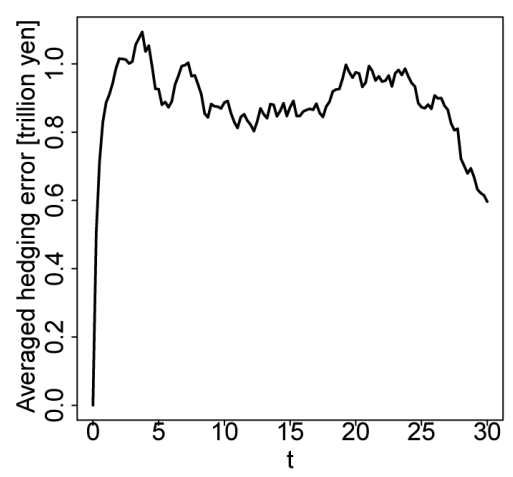

Figure 9. Averaged hedging error $\bar{E}_{t}$.

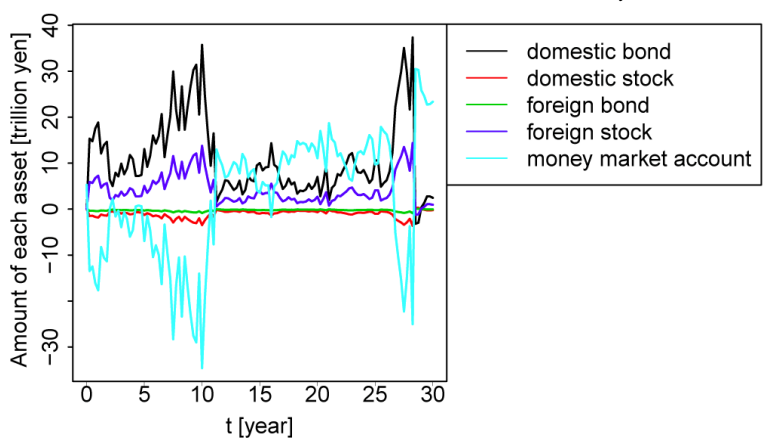

Figure 10. An amount of each asset on the sample path described in Figure 11. The black, red, green, blue and light blue lines represent the amount of a domestic bond, a domestic stock, a foreign bond, a foreign stock and money market account respectively.

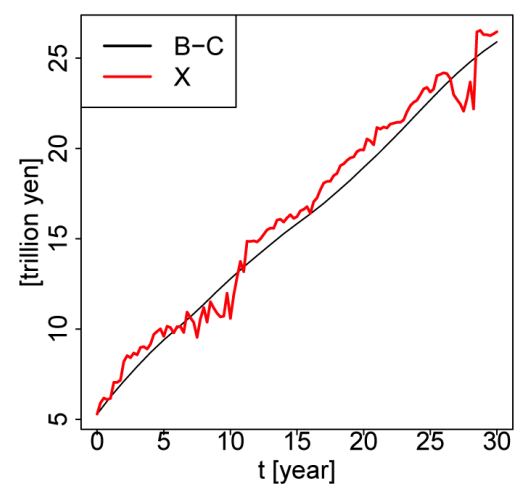

Figure 11. A sample path of $B_{t}-C_{t}$ and $X_{t}$. The black and red lines represent $B_{t}-C_{t}$ and $X_{t}$ respectively.

quite natural.

\section{Summary}

We have proposed a long term portfolio management method which takes into account a liability. The LQG control approach allows us to construct a more suitable long term portfolio strategy than myopic one obtained by the single time period mean variance approach in the sense that we are able to change the strategy at any time. Our optimal portfolio strategy hedges the liability by directly tracking the benchmark process which represents the liability. The strategy is evaluated by the mean square error from the benchmark and hence it is intuitive. Two numerical simulations are served: the former one suggests the situation that our portfolio strategy works well; the latter one provides the result with the empirical data published by Japanese organizations. The result demonstrates that our portfolio strategy is able to hedge the liability, the shortfall of the income of the Japanese welfare pension, over three decades.

This study leaves ample scope for further research. Since our criterion is the mean square error, our portfolio strategy inhibits that our wealth exceeds a liability. This is the similar problem with the traditional mean variance approach. One of approaches to avoid it is that we extend criterion which is able to hedge only the case that our wealth goes under the liability. Then we again face the problem of computability as mentioned in the introductory section.

\section{Acknowledgements}

This work is partially supported by a collaboration research project with the Government Pension Investment Fund in Japan in 2011-2012.

\section{REFERENCES}

[1] The CPP Investment Board, 2013. 
http://www.cppib.ca/Investments/Total_Portfolio_View/

[2] W. H. Fleming and R. W. Rishel, "Deterministic and Stochastic Optimal Control,” Springer-Verlag, New York, 1975. doi:10.1007/978-1-4612-6380-7

[3] G. Deelstra, M. Grasselli and P.-F. Koehl, “Optimal Investment Strategies in the Presence of a Minimum Guarantee," Insurance: Mathematics and Economics, Vol. 33, No. 1, 2003, pp. 189-207. doi:10.1016/S0167-6687(03)00153-7

[4] M. Di Giacinto, S. Federico and F. Gozzi, "Pension Funds with a Minimum Guarantee: A Stochastic Control Approach,” Finance and Stochastics, Vol. 15, No. 2, 2010, pp. 297-342. doi:10.1007/s00780-010-0127-7

[5] F. Menoncin and O. Scaillet, "Optimal Asset Manage- ment for Pension Funds,” Managerial Finance, Vol. 32, No. 4, 2006, pp. 347. doi:10.1108/03074350610652260

[6] R. Gerrard, B. Højgaard and E. Vigna, "Choosing the Optimal Annuitization Time Postretirement," Quantitative Finance, Vol. 12, No. 7, 2012, pp. 1143-1159. doi:10.1080/14697680903358248

[7] The Government Pension Investment Fund in Japan, 2013. http://www.gpif.go.jp/operation/committee/pdf/h191001_ appendix_05.pdf

[8] The Japanese Ministry of Health, Labour and Welfare, 2013.

http://www.mhlw.go.jp/seisakunitsuite/bunya/nenkin/nen kin/zaisei-kensyo/index.html 


\section{Appendix}

\section{Proof of Theorem 1}

The value function corresponding to our problem (5) is defined by

$$
\begin{aligned}
V_{t}(x, y) & =\inf _{\xi \in \mathcal{A}} \mathbb{E}\left[\int_{t}^{T} \gamma_{1}\left(a(s)^{*} Y_{s}-X_{s}^{x_{0}, \xi}\right)^{2} \mathrm{~d} s\right. \\
& \left.+\gamma_{2}\left(A^{*} Y_{T}-X_{T}^{x_{0}, \xi}\right)^{2} \mid X_{t}^{x_{0}, \xi}=x, Y_{t}=y\right] .
\end{aligned}
$$

Hence the corresponding Hamilton-Jacobi-Bellman (HJB) equation is given by

$$
\inf _{\xi \in \mathcal{A}}\left\{\partial_{t} V_{t}(x, y)+\mathscr{L}^{\xi} V_{t}(x, y)+\gamma_{1}\left(a(t)^{*} y-x\right)^{2}\right\}=0,
$$

with terminal condition $V_{T}(x, y)=\gamma_{2}\left(A^{*} y-x\right)^{2}$, $(x, y) \in \mathbb{R} \times \mathbb{R}^{m}$, where $\partial_{t}$ is partial differential operator with respect to $t$ and $\mathscr{L}^{\xi}$ is the infinitesimal generator of the process $\left(X_{t}, Y_{t}\right)$ :

$$
\begin{aligned}
\mathscr{L}^{\xi} \phi(x, y)= & \left(r(t) x+\bar{b}(t)^{*} \xi_{t}\right) \partial_{x} \phi(x, y) \\
& +(\alpha(t) y+h(t))^{*} \partial_{y} \phi(x, y) \\
& +\frac{1}{2}\left[\xi_{t}^{*} \sigma_{S}(t) \sigma_{S}(t)^{*} \xi_{t} \partial_{x}^{2} \phi(x, y)\right. \\
& +2 \xi_{t}^{*} \sigma_{S}(t) \sigma_{Y}(t)^{*} \partial_{y} \partial_{x} \phi(x, y) \\
& \left.+\operatorname{Tr}\left(\sigma_{Y}(t) \sigma_{Y}(t)^{*} \partial_{y}^{2} \phi(x, y)\right)\right],
\end{aligned}
$$

for $\phi \in C^{2}\left(\mathbb{R} \times \mathbb{R}^{m}\right)$. Here $\partial_{x}^{j}$ and $\partial_{y}^{j}$ are the $j$-th order partial differential operators with respect to $x$ and $y$. As $\sigma_{S}(t) \sigma_{S}(t)^{*}$ is positive definite, the infimum of (13) is attained at

$$
\begin{aligned}
\hat{\xi}_{t} & =\frac{-1}{\partial_{x}^{2} V_{t}(x, y)}\left(\sigma_{S}(t) \sigma_{S}(t)^{*}\right)^{-1} \\
& \times\left[\bar{b}(t) \partial_{x} V_{t}(x, y)+\sigma_{S}(t) \sigma_{Y}(t)^{*} \partial_{y} \partial_{x} V_{t}(x, y)\right],
\end{aligned}
$$

and hence (13) can be written as

$$
\begin{aligned}
& \partial_{t} V_{t}(x, y)+r(t) x \partial_{x} V_{t}(x, y)+(\alpha(t) y+h(t))^{*} \partial_{y} V_{t}(x, y) \\
& -\frac{1}{2 \partial_{x}^{2} V_{t}(x, y)} \bar{b}(t)^{*}\left(\sigma_{S}(t) \sigma_{S}(t)^{*}\right)^{-1} \bar{b}(t)\left(\partial_{x} V_{t}(x, y)\right)^{2} \\
& -\frac{\partial_{x} V_{t}(x, y)}{\partial_{x}^{2} V_{t}(x, y)} \\
& \times \bar{b}(t)^{*}\left(\sigma_{S}(t) \sigma_{S}(t)^{*}\right)^{-1} \sigma_{S}(t) \sigma_{Y}(t)^{*} \partial_{y} \partial_{x} V_{t}(x, y) \\
& -\frac{1}{2 \partial_{x}^{2} V_{t}(x, y)} \bar{b}(t)^{*}\left(\sigma_{S}(t) \sigma_{S}(t)^{*}\right)^{-1} \bar{b}(t)\left(\partial_{x} V_{t}(x, y)\right)^{2} \\
& \times\left(\partial_{y} \partial_{x} V_{t}(x, y)\right)^{*} \sigma_{Y}(t) \sigma_{S}(t)^{*} \sigma_{S}(t) \sigma_{Y}(t)^{*} \partial_{y} \partial_{x} V_{t}(x, y) \\
& +c\left(x^{2}-2 x a(t)^{*} y+y^{*} a(t) a(t)^{*} y\right)=0 .
\end{aligned}
$$

Let us try a value function of the form

$$
\begin{aligned}
V_{t}(x, y)= & F^{00}(t) x^{2}+2 x \tilde{F}^{0}(t)^{*} y+y^{*} \tilde{F}(t) y \\
& +G^{0}(t) x+\tilde{G}(t)^{*} y+g(t),
\end{aligned}
$$

where $F^{00}, G^{0}, g:[0, T] \rightarrow \mathbb{R}, \tilde{F}^{0}, \tilde{G}:[0, T] \rightarrow \mathbb{R}^{m}$ and $\tilde{F}$ is a time-dependant symmetric matrix. It is straightforward to see that

$$
\left\{\begin{array}{l}
\frac{\mathrm{d}}{\mathrm{d} t} F^{00}(t)+c+2 r(t) F^{00}(t) \\
-\bar{b}(t)^{*}\left(\sigma_{S}(t) \sigma_{S}(t)^{*}\right)^{-1} \bar{b}(t) F^{00}(t)=0, \\
F^{00}(T)=C,
\end{array}\right.
$$

$$
\left\{\begin{array}{l}
\frac{\mathrm{d}}{\mathrm{d} t} \tilde{F}^{0}(t)-c a(t)+r(t) \tilde{F}^{0}(t)+\alpha(t)^{*} \tilde{F}^{0}(t) \\
-\bar{b}(t)^{*}\left(\sigma_{S}(t) \sigma_{S}(t)^{*}\right)^{-1} \bar{b}(t) \tilde{F}^{0}(t)=0, \\
\tilde{F}^{0}(T)=-2 C A,
\end{array}\right.
$$

$$
\left\{\begin{array}{l}
\frac{\mathrm{d}}{\mathrm{d} t} G^{0}(t)+r(t) G^{0}(t)+2 h(t)^{*} \tilde{F}^{0}(t) \\
-\bar{b}(t)^{*}\left(\sigma_{S}(t) \sigma_{S}(t)^{*}\right)^{-1}(b(t)-r(t) \mathbf{1}) G^{0}(t) \\
-\bar{b}(t)^{*}\left(\sigma_{S}(t) \sigma_{S}(t)^{*}\right)^{-1} \sigma_{S}(t) \sigma_{Y}^{*}(t) \tilde{F}^{0}(t)=0, \\
G^{0}(T)=0,
\end{array}\right.
$$

$$
\left\{\begin{array}{l}
\frac{\mathrm{d}}{\mathrm{d} t} \tilde{F}(t)+c a(t) a(t)^{*}+2 \alpha(t)^{*} \tilde{F}(t) \\
-\frac{1}{F^{00}(t)} \tilde{F}^{0}(t) \tilde{F}^{0}(t)^{*}=0, \\
\tilde{F}(T)=C A A^{*},
\end{array}\right.
$$

$$
\left\{\begin{array}{l}
\frac{\mathrm{d}}{\mathrm{d} t} \tilde{G}(t)+r(t) \tilde{G}(t)+\alpha(t)^{*} \tilde{G}(t)+2 h(t)^{*} \tilde{F}(t) \\
-\frac{G^{0}(t)}{F^{00}(t)} \bar{b}(t)^{*}\left(\sigma_{S}(t) \sigma_{S}(t)^{*}\right)^{-1} \bar{b}(t) G^{0}(t) \\
-\frac{\tilde{F}^{0}(t)}{F^{00}(t)} \bar{b}(t)^{*}\left(\sigma_{S}(t) \sigma_{S}(t)^{*}\right)^{-1} \\
\times\left(\sigma_{S}(t) \sigma_{Y}^{*}(t) \tilde{F}^{0}(t)\right)=0 \\
\tilde{G}(T)=0
\end{array}\right.
$$




$$
\left\{\begin{array}{l}
\frac{\mathrm{d}}{\mathrm{d} t} g(t)+h(t) \tilde{G}(t)+\operatorname{Tr}\left(\sigma_{Y} \sigma_{Y}^{*} \tilde{F}(t)\right) \\
-\frac{\left(G^{0}(t)\right)^{2}}{4 F^{00}(t)} \bar{b}(t)^{*}\left(\sigma_{S}(t) \sigma_{S}(t)^{*}\right)^{-1} \bar{b}(t) \\
-\frac{G^{0}}{2 F^{00}(t)} \bar{b}(t)^{*}\left(\sigma_{S}(t) \sigma_{S}(t)^{*}\right)^{-1} \\
\times \sigma_{S}(t) \sigma_{Y}^{*}(t) \tilde{F}^{0}(t)=0, \\
g(T)=0,
\end{array}\right.
$$

and then the associated candidate strategy is represented by

$$
\begin{aligned}
\hat{\xi}_{t}= & \frac{-1}{2 F^{00}(t)}\left(\sigma_{S}(t) \sigma_{S}(t)^{*}\right)^{-1} \\
& \times\left[\bar{b}(t)\left(2 F^{00}(t)+2 \tilde{F}^{0}(t)+G^{0}(t)\right)\right. \\
& \left.+2 \sigma_{S}(t) \sigma_{Y}(t)^{*} \tilde{F}^{0}(t)\right] .
\end{aligned}
$$

Since (14) and (15) are linear ODEs, they have unique solutions on $[0, T]$. The existence of unique $\tilde{F}^{0}(t)$ suggests that (16) and (17) are linear ODEs. Hence (16) and (17) also have unique solutions on $[0, T]$. In the same manner, the existence of unique solutions of (18) and (19) are guaranteed. Therefore $\hat{\xi}_{t} \in \mathcal{A}$ since $X_{t}$ and $Y_{t}$ in $\mathcal{L}^{2}\left(\mu_{T} \times \mathbb{P}\right)$.

We now start the verification, i.e., we show that $V_{0}\left(x_{0}, y_{0}\right) \leq J[\xi], \quad \xi \in \mathcal{A}$ and $V_{0}\left(x_{0}, y_{0}\right)=J[\hat{\xi}]$. To this end, we introduce a sequence of stopping times $\left\{\tau_{l}\right\}_{l \in \mathbb{N}}$ s.t.

$$
\tau_{l}=\inf \left\{u \geq 0: \int_{0}^{u}\left|X_{t} \xi_{t}\right|^{2} \mathrm{~d} t \geq l, \int_{0}^{u}\left|Y_{t} \xi_{t}\right|^{2} \mathrm{~d} t \geq l\right\} .
$$

Applying the Ito formula to $V_{T \wedge \tau_{l}}\left(X_{T \wedge \tau_{l}}, Y_{T \wedge \tau_{l}}\right)$ and taking expectation, we have

$$
\begin{aligned}
V_{0}( & \left.x_{0}, y_{0}\right) \\
=\mathbb{E} & {\left[\int_{0}^{T \wedge \tau_{l}}\left(-\partial_{t} V_{t}\left(X_{t}, Y_{t}\right)-L^{\xi} V_{t}\left(X_{t}, Y_{t}\right)\right) \mathrm{d} t\right.} \\
& \left.+V_{T}\left(X_{T \wedge \tau_{l}}, Y_{T \wedge \tau_{l}}\right)\right] \\
-\mathbb{E} & {\left[\int_{0}^{T \wedge \tau_{l}} \partial_{x} V_{t}\left(X_{t}, Y_{t}\right) \xi^{*} \sigma_{S}(t) \mathrm{d} W_{t}\right] } \\
-\mathbb{E} & {\left[\int_{0}^{T \wedge \tau_{l}}\left(\partial_{x} V_{t}\left(X_{t}, Y_{t}\right)\right)^{*} \sigma_{Y}(t) \mathrm{d} W_{t}\right] . }
\end{aligned}
$$

As $X_{t}$ and $Y$ are in $\mathcal{L}^{2}\left(\mu_{T} \times \mathbb{P}\right)$ and $\sigma_{Y}, \quad \tilde{F}^{0}$, $\tilde{F}$ and $\tilde{G}$ are continuous functions on $[0, T]$, the last term vanishes:

$$
\begin{aligned}
& \mathbb{E}\left[\int_{0}^{T \wedge \tau_{l}}\left(\partial_{y} V_{t}\left(X_{t}, Y_{t}\right)\right)^{*} \sigma_{Y}(t) \mathrm{d} W_{t}\right] \\
& =2 \mathbb{E}\left[\int_{0}^{T \wedge \tau_{l}} X_{t} \tilde{F}^{0}(t)^{*} \sigma_{Y}(t) \mathrm{d} W_{t}\right] \\
& +2 \mathbb{E}\left[\int_{0}^{T \wedge \tau_{l}} Y_{t}^{*} \tilde{F}(t) \sigma_{Y}(t) \mathrm{d} W_{t}\right] \\
& +\mathbb{E}\left[\int_{0}^{T \wedge \tau_{l}} \tilde{G}(t)^{*} \sigma_{Y}(t) \mathrm{d} W_{t}\right]=0 .
\end{aligned}
$$

By the definition of $\tau_{l}$, the continuity of the functions $F^{00}, \tilde{F}^{0}$ and $G^{0}$, and the fact that $\xi \in \mathcal{A}$, the remaining stochastic integral term also vanishes:

$$
\begin{aligned}
& \mathbb{E}\left[\int_{0}^{T \wedge \tau_{l}} \partial_{x} V_{t}\left(X_{t}, Y_{t}\right) \xi_{t}^{*} \sigma_{S}(t) \mathrm{d} W_{t}\right] \\
& =2 \mathbb{E}\left[\int_{0}^{T \wedge \tau_{l}} F^{00}(t) X_{t} \xi_{t}^{*} \sigma_{S}(t) \mathrm{d} W_{t}\right] \\
& +2 \mathbb{E}\left[\int_{0}^{T \wedge \tau_{l}} \tilde{F}^{0}(t)^{*} Y_{t} \xi_{t}^{*} \sigma_{S}(t) \mathrm{d} W_{t}\right] \\
& +\mathbb{E}\left[\int_{0}^{T \wedge \tau_{l}} G^{0}(t) \xi_{t}^{*} \sigma_{S}(t) \mathrm{d} W_{t}\right] \\
& =0 .
\end{aligned}
$$

Since $\tau_{l} \nearrow \infty$ when $l$ goes to infinity, we get

$$
\begin{aligned}
& V_{0}\left(x_{0}, y_{0}\right) \\
& =\mathbb{E}\left[\int_{0}^{T}\left(-\partial_{t} V_{t}\left(X_{t}, Y_{t}\right)-L^{\xi} V_{t}\left(X_{t}, Y_{t}\right)\right) \mathrm{d} t\right. \\
& \left.+V_{T}\left(X_{T}, Y_{T}\right)\right] .
\end{aligned}
$$

By the HJB Equation (13) and its terminal condition, we obtain

$$
V_{0}\left(x_{0}, y_{0}\right) \leq \mathbb{E}\left[\int_{0}^{T} \gamma_{1}\left(a(t)^{*} y-x\right)^{2} \mathrm{~d} t+\gamma_{2}\left(A^{*} y-x\right)^{2}\right],
$$

which means that $V_{0}\left(x_{0}, y_{0}\right) \leq J[\xi], \xi \in \mathcal{A}$. In the same manner we find that $V_{0}\left(x_{0}, y_{0}\right)=J[\hat{\xi}]$ and then the claim is established. 